MOTHS OF WESTERN NORTH AMERICA 
This page intentionally left blank 


\section{Moths of Western North America}

JERRY A. POWELL

PAUL A. OPLER 
University of California Press, one of the most distinguished university presses in the United States, enriches lives around the world by advancing scholarship in the humanities, social sciences, and natural sciences. Its activities are supported by the UC Press Foundation and by philanthropic contributions from individuals and institutions. For more information, visit www.ucpress.edu.

University of California Press

Berkeley and Los Angeles, California

University of California Press, Ltd.

London, England

\section{(C) 2009 by The Regents of the University of California}

Photo/image credits: T. Arcand: 61.25-61.27, 61.31, 61.34, 62.10, $62.20,62.23,62.24,63.12,63.31,63.36,64.1,64.24-64.26,64.29$ E. Buckner-Opler: 63.3, 63.5, 63.21, 63.28-63.30. California Department of Food \& Agriculture: 61.6. Canadian National Collection (CNC): 61.28, 61.32, 62.13, 62.25, 62.32, 63.19, 63.20, 63.26, 63.27, $63.33,63.35,64.3-64.6,64.9,64.13,64.20-64.23,64.27,64.28$, 64.32-64.34. R. Coville: Last page color section, 59.35, 60.4, 60.5 60.7, 60.17, 60.21-60.24, 60.27, 61.2. H. V. Daly: 60.1. L. Dyer: 61.7. D. C. Ferguson: $61.11,61.29,61.30,61.33,61.36,62.2,62.3$, 62.5-62.8, 62.11, 62.14, 62.16, 62.26, 62.27, 62.32, 62.34, 64.7, 64.8, 64.12, 64.14-64.16. C. Hansen: 61.13. P. G. Johnson II: First page color section, $59.2,60.6,60.9,60.19,60.30,62.34,63.22-63.25$

N. McFarland: 61.9. L. Minor-Penland: 61.13. P. A. Opler: 61.23, 63.8, 63.9, 64.10, 64.11. S. Passoa: 64.19. D. J. Powell: 59.25, 59.33, 60.10, $60.15,61.4,61.5,61.14,61.15-61.22$. J. A. Powell: 59.3, 59.4-59.22, $59.26-59.32,59.34,59.36,60.2,60.3,60.8,60.11-60.16,60.18,60.20$ $60.25,60.26,60.28,60.29,60.31-60.36,61.1,61.3,61.24,62.33,63.2$, $64.17,64.18,64.35$. E. S. Ross 59.23. B. Scaccia 61.35, 62.1, 62.4, 62.9, 62.12, 62.15, 62.17-62.19, 62.21, 62.22. D. L. Wagner 59.1, 61.8, 61.10. B. Walsh 62.28-62.31, 62.35, 62.36, 63.1, 63.4, 63.6, 63.7, $63.10,63.11,63.13-63.18,64.2,64.36$.
Library of Congress Cataloging-in-Publication Data

Powell, Jerry A.

Moths of western North America / Jerry A. Powell, Paul A. Opler. p. $\mathrm{cm}$.

Includes bibliographical references and index.

ISBN 978-0-520-25197-7 (case : alk. paper) 1. Moths-West (U.S.)

2. Moths-Northwest, Canadian. I. Opler, Paul A. II. Title.

QL548.P69 2009

$595.780970-\mathrm{dc} 22$

2008048605

Manufactured in China.

$\begin{array}{lllllllll}17 & 16 & 15 & 14 & 13 & 12 & 11 & 10 & 09\end{array}$

$\begin{array}{llllllllll}10 & 9 & 8 & 7 & 6 & 5 & 4 & 3 & 2 & 1\end{array}$

The paper used in this publication meets the minimum requirements of ANSI/NISO Z 39.48-1992 (R 1997)(Permanence of Paper).

Cover: Arctia caja (Linnaeus), California, Sierra Co., San Francisco State U., Sierra Nevada Field Campus, July 2007. Species is also shown in plate 47.8, which is from Elko Co., Nevada. The Sierra Nevada population moths differ in color and pattern from the Rocky Mountain and Basin Ranges form. Photograph by Rollin Coville. 